\title{
A Protective Effect against Undesirable Increase of Dihydroetorphine Permeation through Damaged Skin by Using Pressure-Sensitive Adhesive Tape with an Ethylene-Vinyl Acetate Co-Polymer Membrane
}

\author{
Satoshi Ohmori, Yohei Sugryama, and Yasunori Morimoto* \\ Faculty of Pharmaceutical Sciences, Josai University, 1-1 Keyakidai, Sakado, Saitama 350-0295, Japan. \\ Received August 30, 2000; accepted October 18, 2000
}

\begin{abstract}
The release kinetics of dihydroetorphine (DHE) from pressure-sensitive adhesive (PSA) tape with an ethylene-vinyl acetate co-polymer (EVA) membrane as a diffusion-controlling membrane and its protective effect from an unpredictable increase in skin permeation of DHE caused by stratum corneum damage were investigated. The DHE flux through the EVA membrane was enhanced with the increase of vinyl acetate content. Although the DHE release from the PSA tape was proportional to the square root of the time, the release from the PSA tape covered with the EVA membrane was dominated by zero-order rate. The release rate increased by the addition of isopropyl myristate to the PSA layer, due to the increase of solubility and diffusivity of DHE in the PSA layer, and not a decrease of permeation resistance in the EVA membrane. When using the PSA tape with the EVA membrane, the steady-state flux of DHE through hairless rat skin with stratum corneum damage was not 2-fold more than that through non-damaged skin. Plasma DHE concentration rose promptly above $5 \mathrm{ng} / \mathrm{ml}$ after the application of the PSA tape onto the damaged skin in hairless rat. In contrast, when the PSA tape with the EVA membrane was applied onto the damaged or non-damaged skin, plasma concentrations in the both cases were maintained in the therapeutic range $(0.2-1.2 \mathrm{ng} / \mathrm{ml})$. These results suggest that the PSA tape with the EVA membrane can be used to protect from the unpredictable increase in skin permeation of DHE due to stratum corneum damage.
\end{abstract}

Key words dihydroetorphine; ethylene-vinyl acetate co-polymer; diffusion controlling membrane; damaged skin; pressure sensitive adhesive; hairless rat

Dihydroetorphine, 7,8-dihydro-7 $\alpha-(-[1-(R)$-hydroxy-1methylbutyl]-6,14-endo-ethanotetrahydro-oripavine (DHE), is a selective $\mu$-opioid receptor agonist. ${ }^{1)}$ DHE produces an extraordinarily strong analgesia, which is $1000-12000$-fold more potent than morphine. ${ }^{2-4)}$ In a previous study, we demonstrated that DHE was quickly distributed into the brain and produces a potent analgesia, and then DHE was rapidly eliminated with a short half-life, $37.7 \mathrm{~min}$, in hairless rat. ${ }^{5}$ After oral administration DHE had a poor bioavailability, below $1 \%$ of dose, because of an extensive first-pass metabolism as a glucuronide form. In contrast after intracutaneous injection, DHE was well absorbed and quickly produced potent analgesic effect. Therefore, we investigated the transdermal delivery of DHE and have confirmed that topical application of DHE using pressure sensitive adhesive (PSA) tape can produce a continuous analgesia for $24 \mathrm{~h}$ in hairless rat. ${ }^{6}$

The outer layer of the skin, the stratum corneum (SC), is a significant barrier for the invasion of substances in the environment and for the delivery of many drugs. ${ }^{7)}$ The permeation resistance of DHE in hairless rat skin occurs in the SC, especially in vivo condition. ${ }^{6}$ Therefore, the flux of DHE for SC stripped skin was much higher than that for intact skin. This result suggests that if the SC is damaged such as by a skin wound, irritation by chemical substance, dermatological illness or atopy like allergic reaction, ${ }^{8-11)}$ DHE will excessively permeate the skin and the plasma DHE concentration maybe increase above the therapeutic range. If the topically applied device has a release-controlling membrane, it is possible to prevent such an unpredictable increase in DHE permeation through the skin.

There are many reports regarding topically applied devices with the ethylene-vinyl acetate co-polymer (EVA) membrane as the diffusion-controlling membrane. ${ }^{12-14)}$ EVA membrane has been used in several release-controlling devices, because of its safety and the ease to modify the permeability by adjusting the vinyl acetate content. ${ }^{15)}$ In this study, we used the EVA membrane for control of the release rate of DHE from the PSA layer. The aims of this study are to select suitable concentrations of vinyl acetate in the EVA membrane and of isopropyl myristate (IPM) in the PSA layer by kinetic analysis for the release profiles of DHE and to evaluate the protective effect for the excessive increase of plasma DHE concentration during the application onto SC damaged skin.

\section{MATERIALS AND METHODS}

Chemicals DHE was synthesized from codeine using reported procedures. ${ }^{2,16)}$ Styrene-isoprene-styrene block copolymer (Cariflex TR-1107) was purchased from Shell Chemical Co. (Tokyo, Japan). Rosin ester (KE-311) was purchased from Arakawa Chemical Co. (Osaka, Japan). Various contents of vinyl acetate of EVA (EVAFLEX ${ }^{\circledR}$ ) were kindly supplied by Mitsui Du Pont Polychemical Co. (Tokyo, Japan). IPM and $p$-hydroxybenzoic acid methyl ester were purchased from Tokyo Chemical Industry Co. (Tokyo, Japan). Buprenorphine hydrochloride was kindly supplied by Otsuka Pharmaceuticals (Tokyo, Japan). All other reagents were of analytical grade.

Animals Male hairless rats (WBN/ILA-Ht strain) weighing 250 to $280 \mathrm{~g}$ ( $11-13$ weeks old) used in all experiments were supplied by the Life Science Research Center of Josai University (Saitama, Japan) and the Ishikawa Experimental Animal Laboratory (Saitama, Japan). The animals had free access to a standard rodent diet and clean drinking water.

Preparation of the PSA Tape The PSA tape was prepared according to our previous report. $\left.{ }^{6}\right)$ Briefly, DHE, 
styrene-isoprene-styrene block co-polymer, rosin ester and IPM were dissolved in chloroform. The mixture was cast onto polyethylene terephthalate film and then dried. The PSA tape was covered with a release liner and then the tape was pulled out circularly. The thickness of the layer containing DHE was $65 \mu \mathrm{m}$. DHE content was $61 \mu \mathrm{g} / \mathrm{cm}^{2}$ and the most of DHE had been dispersed in the tape when observed under a light microscope (Fig. 1). The IPM concentrations were prepared as 0, 5, 10 and 20\%. Separately, a DHE-absent PSA layer was prepared using the same procedure but excluding the DHE.

Preparation of the EVA Membrane The EVA membrane was prepared by the hot-melt method. ${ }^{17)}$ EVA beads containing 14, 19, 28 and $33 \%$ of vinyl acetate were pressed to a thickness of $50 \mu \mathrm{m}$ at $100{ }^{\circ} \mathrm{C}$ using Test Press (Tester Sangyo Co. Ltd., Tokyo, Japan). Immediately before use, the EVA membrane was placed on the DHE containing PSA tape.

Determination of the DHE Solubility in the PSA Tape The EVA membrane was placed between the PSA tape containing dispersed DHE and a DHE-absent PSA layer. DHE permeated into the EVA membrane and the PSA layer until the DHE concentration in each layer were saturated. After being left for $72 \mathrm{~h}$ at ambient temperature, the PSA layer was peeled off, and then it was immersed in methanol in order to recover the DHE dissolved in this layer. After a 24-h incubation at $37^{\circ} \mathrm{C}$, the DHE concentration in the methanol was measured.

Skin Preparation Abdominal region of the rats were carefully shaved, and the skin excised under diethylether anesthesia. Subcutaneous tissue was removed from the skins. In preparation of SC damaged skin, SC was striped from the skin by stripping 20 times with adhesive cellophane tape (Nichiban Co., Tokyo, Japan) before excision of the skin.

In-Vitro Permeation or Release Studies In the study for the permeation of DHE through the EVA membrane, the EVA membrane was inserted between two halves of side-byside diffusion cells encompassed by a $37^{\circ} \mathrm{C}$ water jacket with a volume of $2.5 \mathrm{ml}$ and a $0.95 \mathrm{~cm}^{2}$ effective diffusion area. DHE suspension and distilled water were filled into the donor and receiver compartments of the diffusion cell, respectively, and both compartments were stirred with a magnetic-bar driven by a constant-speed synchronous motor (Scinics, Tokyo, Japan). In the study for the release of DHE from the PSA tape, the PSA tape was attached to one half of the side-by-side diffusion cells and then distilled water was filled into the receiver compartment of the diffusion cell. In the study of the permeation of DHE through the skin, the dermis side of the skin was attached to one half of the sideby-side diffusion cells. The PSA tape was applied to the SC side of the skin, and then distilled water was filled into the receiver compartment of the diffusion cell. In all studies, the receiver solution was withdrawn at appropriate times for measurement of the permeated or released drug and replaced with fresh distilled water.

In-Vivo Application of PSA Tape on the Skin The abdominal region of hairless rats was carefully shaved and wiped, in order to the abdominal application of PSA tape. In the case of application to the SC damaged skin, the abdominal skin was prepared by stripping 20 times with adhesive cellophane tape under diethylether anesthesia. Under light anesthesia, PSA tape with the EVA membrane $\left(0.95 \mathrm{~cm}^{2}\right)$ or PSA tape without the EVA membrane $\left(0.28 \mathrm{~cm}^{2}\right)$ was applied to the abdominal skin of the rats. If the rats were sedated by the pharmacological effect of DHE, the diethylether anesthesia was discontinued. Blood samples were withdrawn from the jugular vein. Plasma was separated by centrifugation and stored at $-20^{\circ} \mathrm{C}$ until analysis.

Measurement of DHE Concentrations in the Receiver Solution and the Methanol One hundred microliters of the sample were mixed with an equal volume of $p$-hydroxybenzoic acid methyl ester as an internal standard $(10 \mu \mathrm{g} / \mathrm{ml}$ in acetonitrile). After $9000 \times \boldsymbol{g}$ centrifugation, an aliquot of the supernatant $(20 \mu \mathrm{l})$ was applied to a high performance liquid chromatography system (LC-10A, Shimadzu Co., Kyoto, Japan). Phosphate buffer $(1 / 30 \mathrm{M}, \mathrm{pH} 5.2)$-acetonitrile-methanol $(70: 21: 9)$ as a mobile phase was delivered at $1.0 \mathrm{ml} / \mathrm{min}$ through Nucleosil $5 \mathrm{C}_{18}(4.6 \mathrm{~mm}$ i.d. $\times 250 \mathrm{~mm}$, Macherey Nagel, Germany) under $40^{\circ} \mathrm{C}$. DHE and the internal standard were detected at $215 \mathrm{~nm}$.

Measurement of DHE Concentrations in Rat Plasma Plasma DHE concentrations were measured by liquid chromatography-tandem mass spectrometry. ${ }^{18)}$ Briefly, an exact volume less than $0.5 \mathrm{ml}$ of the sample was mixed with buprenorphine as an internal standard and $50 \mathrm{~mm}$ phosphate buffer (pH 6.0). The mixture was applied to a Bond Elut Certify ${ }^{\circledR}$ cartridge $(3 \mathrm{ml} / 130 \mathrm{mg}$, Varian, Harbor City, CA, U.S.A.), and the cartridge was washed with $100 \mathrm{~mm}$ acetic acid and methanol. The eluent with $2 \%$ ammonium hydroxide in ethyl acetate was collected and redissolved in acetonitrile-water $(80: 20)$, and an aliquot was applied to a LC-MSMS system (API-300 ${ }^{\circledR}$, Perkin Elmer-SCIEX, Foster City, CA, U.S.A.). Inertsil ODS-2 $(5 \mu \mathrm{m}, 2.1 \mathrm{~mm}$ i.d. $\times 150 \mathrm{~mm}$, GL Science, Tokyo, Japan) filled with acetonitrile- $50 \mathrm{~mm}$ ammonium acetate $(95: 5)$ was used. DHE and the internal standard were detected at $m / z$ 414/414 and 468/468, respectively. The limit of quantitation with acceptance criteria as intra- and inter-assay precision within $20 \%$ was $0.05 \mathrm{ng} / \mathrm{ml}$ in plasma.

Calculation of Kinetic Parameters Figure 2 shows the scheme of the PSA tape with or without the EVA membrane and of the mathematical model for the release of DHE. Type I (Fig. 2A) is a mono-layer tape as the PSA layer contained DHE. Type II (Fig. 2B) is a bi-layer tape as the PSA layer attached with the EVA membrane. Type III (Fig. 2C) is a trilayer tape as the PSA layer attached with the EVA membrane and the adhesive layer. It was postulated that the DHE concentrations in the PSA tape $\left(C_{1}\right)$, EVA membrane $\left(C_{2}\right)$ and adhesive layer $\left(C_{3}\right)$ at a position $x$ and at time $t$ followed by the Fick's Second-Law as follows:

$$
\begin{aligned}
& \partial C_{1} / \partial t=D_{1} \cdot \partial^{2} C_{1} / \partial x^{2} \\
& \partial C_{2} / \partial t=D_{2} \cdot \partial^{2} C_{2} / \partial x^{2} \\
& \partial C_{3} / \partial t=D_{3} \cdot \partial^{2} C_{3} / \partial x^{2}
\end{aligned}
$$

where $D_{1}, D_{2}$ and $D_{3}$ are the diffusivity in each layer.

In the case of Type I, the initial and boundary conditions were set as follows:

$$
\begin{aligned}
& \text { at } t=0, \quad C_{1}=C_{\mathrm{S}} \\
& \text { at } t>0 \text { and } x=L_{1}, \quad C_{1}=0
\end{aligned}
$$

where $C_{\mathrm{S}}$ is the solubility in the PSA matrix and $L_{1}$ is the thickness of the PSA layer. It was postulated that the dissolu- 
tion rate of DHE was much higher than the diffusion in the PSA matrix, and so the amount of DHE lost by its diffusion in the matrix would be immediately compensated by the dissolution of dispersed DHE. ${ }^{19)}$ Therefore, when the sum of dissolved $\left(C_{1}\right)$ and undissolved concentration $\left(C_{1}^{\prime}\right)$ is greater than $C_{\mathrm{S}}$, in the next moment, $C_{1}$ and $C_{1}^{\prime}$ are replaced by $C_{\mathrm{S}}$ and $\left(C_{1}+C_{1}^{\prime}\right)-C_{\mathrm{S}}$, respectively. If the sum of $C_{1}$ and $C_{1}^{\prime}$ is smaller than or equal to $C_{\mathrm{S}}, C_{1}$ and $C_{1}^{\prime}$ are replaced by $C_{1}+C_{1}^{\prime}$ and zero, respectively.

In the case of Type II, the initial and boundary conditions were set as follows:

$$
\begin{aligned}
& \text { at } t=0, \quad C_{1}=C_{\mathrm{S}} \quad \text { and } \quad C_{2}=0 \\
& \text { at } t>0 \text { and } x=L_{1}, \quad C_{2}=K_{2 / 1} \cdot C_{1} \text { and } D_{1} \cdot d C_{1} / d x=D_{2} \cdot d C_{2} / d x \\
& \text { at } t>0 \text { and } x=L_{1}+L_{2}, \quad C_{2}=0
\end{aligned}
$$

where $L_{2}$ is the thickness of the EVA membrane and $K_{2 / 1}$ is the partition coefficient of DHE between the EVA membrane and the PSA layer.

In the case of Type III, initial and boundary conditions were set as follows:

$$
\begin{aligned}
& \text { at } t=0, \quad C_{1}=C_{\mathrm{S}} \text { and } C_{2}, C_{3}=0 \\
& \text { at } t>0 \text { and } x=L_{1}, \quad C_{2}=K_{2 / 1} \cdot C_{1} \text { and } D_{1} \cdot d C_{1} / d x=D_{2} \cdot d C_{2} / d x \quad \text { (2C) } \\
& \text { at } t>0 \text { and } x=L_{1}+L_{2}, \quad C_{3}=K_{3 / 2} \cdot C_{2} \text { and } D_{2} \cdot d C_{2} / d x=D_{3} \cdot d C_{3} / d x
\end{aligned}
$$$$
\text { at } t>0 \text { and } x=L_{1}+L_{2}+L_{3}, \quad C_{3}=0
$$

where $L_{3}$ is the thickness of the adhesive layer and $K_{3 / 2}$ is the partition coefficients of DHE between the adhesive layer and the EVA membrane. If the adhesive layer is composed of the same element of the PSA layer, $D_{3}$ and $K_{3 / 2}$ are replaced by $D_{1}$ and the inverse of $K_{2 / 1}$, respectively.

In each case, the release rate of DHE from the last layer into the receiver compartment $(J)$ and the cumulative amount of released DHE $(Q)$ is represented as follows:

$$
\begin{aligned}
& J=-D_{n}\left(d C_{n} / d x\right), \quad n=1,2,3 \text { and } x=L_{1}, L_{1}+L_{2}, L_{1}+L_{2}+L_{3}, \\
& \quad \text { respectively } \\
& Q=\sum_{0}^{t}(J \times t)
\end{aligned}
$$

Partial differential Eq. 1, and ordinary differential Eqs. 3B, $3 \mathrm{C}$ and $4 \mathrm{C}$, are approximated by the explicit finite-difference equation. ${ }^{20)}$ The number of spatial mesh was 20 in the PSA tape layer, which satisfied the degree of accuracy in this study, and was 10 in the EVA membrane and the adhesive layer. The cumulative amount of released DHE was fitted to Eq. 7 by the weighted least-square method using a quasiNewton algorithm on the solver-function of Microsoft Excel $^{\circledR} 97$.

Initially, the cumulative amount released from Type I was fitted to Eq. 7 using fixed $L_{1}$ and $C_{\mathrm{S}}$, and thereby $C_{1}^{\prime}$ and $D_{1}$ were estimated. Initial values of $C_{1}^{\prime}$ and $D_{1}$ for fitting-calculation were estimated by visual inspection in Fig. 3. Secondary, the cumulative amount released from Type II was fitted to Eq. 7 using fixed $C_{\mathrm{S}}, C_{1}^{\prime}, L_{1}, L_{2}$ and $D_{1}$, and thereby $K_{2 / 1}$ and $D_{2}$ were estimated. Initial values of $K_{2 / 1}$ and $D_{2}$ for fitting-calculation were estimated by visual inspection in Fig. 6A. Thirdly, the cumulative amount released from Type III was simulated by Eq. 7 using fixed $C_{\mathrm{S}}, C_{1}^{\prime}, L_{1}, L_{2}, L_{3}, D_{1}, D_{2}$ and $K_{2 / 1}$.

The permeation resistance of DHE in the EVA membrane

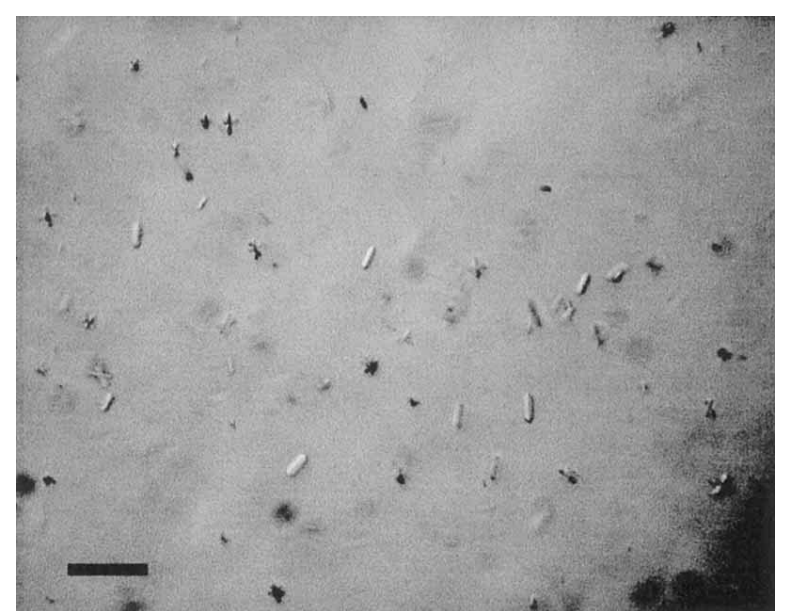

Fig. 1. Microscopic Observation of the PSA Tape with $10 \%$ of IPM Hatched bar indicates $20 \mu \mathrm{m}$.

$\left(R_{2}\right)$ and the adhesive layer $\left(R_{3}\right)$ were calculated as follows:

$$
\begin{aligned}
& R_{2}=L_{2} /\left(D_{2} \cdot K_{2 / 1}\right) \\
& R_{3}=L_{3} /\left(D_{3} \cdot K_{2 / 1} \cdot K_{3 / 2}\right)
\end{aligned}
$$

The permeation resistance of DHE in the excised hairless rat skin $\left(R_{\text {skin }}\right)$ in the in-vitro permeation study was calculated using the solubility of DHE in the PSA tape $\left(C_{\mathrm{S}}\right)$ and the steady-state flux $\left(J_{\mathrm{SS}}\right)$ as follows:

$$
R_{\text {skin }}=C_{\mathrm{S}} / J_{\mathrm{SS}}
$$

\section{RESULTS AND DISCUSSION}

Release of DHE from the PSA Tape, Type I Needlelike crystals of DHE in the PSA tape with $10 \%$ of IPM were clearly observed under the microscope (Fig. 1). The solubility of DHE in the PSA tape with 0,5, 10 and 20\% of IPM was $0.722,1.18,2.20$ and $2.98 \mathrm{mg} / \mathrm{cm}^{3}$, respectively (Table 1). These results indicate that $91.3,85.7,73.4$ and $63.9 \%$ of the DHE included $\left(9.38 \mathrm{mg} / \mathrm{cm}^{3}\right)$ had crystallized in the PSA tape with $0,5,10$ and 20\% IPM, respectively. The cumulative amount of released DHE from the PSA tape, Type I, during the initial phase was proportional to the square of time as reported by Higuchi, ${ }^{19)}$ and its slope increased with the IPM content (Fig. 3). Calculated parameters, $D_{1}$ and $C_{1}^{\prime}$, by fitting-calculation did not depend on their initial values. In the case of the tape with 0 and 5\% IPM, the initial values of $C_{1}^{\prime}$ were substituted by calculated $C_{1}^{\prime}$ of the tape with $10 \%$ IPM, because the cumulative amount of released DHE from the tape with 0 and 5\% IPM did not reached plateau level. As the results of the calculation, the diffusivities of DHE in the PSA layer, $D_{1}$, increased with the IPM content, more extensively than the increase of the solubility (Table 1). Undesirably, the total amounts of DHE released from the PSA tape with 0, 5, 10 and $20 \%$ IPM, which were estimated using $L_{1}, C_{1}^{\prime}$ and $C_{\mathrm{S}}$, were $36.3,42.9,48.0$ and $53.8 \mu \mathrm{g} / \mathrm{cm}^{2}$ equivalent to 59.5 , $70.3,78.7$ and $88.2 \%$ of the included DHE $\left(61 \mu \mathrm{g} / \mathrm{cm}^{2}\right)$, respectively. It is suggested that a part of dispersed DHE remained in the PSA tape and decreased with the increase of IPM content. From these results, IPM is required to adequately release DHE from the PSA tape.

Influence of IPM on the Permeation of DHE through 
Table 1. Effect of IPM Content in the PSA Layer on the Kinetic Parameters for the Diffusion of DHE into the PSA Layer, the EVA Membrane, the Adhesive Layer and the Hairless Rat Skin

\begin{tabular}{|c|c|c|c|c|c|c|c|c|c|c|c|c|c|}
\hline \multirow{2}{*}{$\begin{array}{c}\text { IPM } \\
\text { content } \\
\text { in PSA } \\
\text { layer } \\
(\%)\end{array}$} & \multicolumn{4}{|c|}{ PSA layer ${ }^{a)}$} & \multicolumn{4}{|c|}{ EVA membrane $^{b)}$} & \multicolumn{4}{|c|}{ Adhesive layer ${ }^{c)}$} & \multirow{2}{*}{$\begin{array}{c}\begin{array}{c}\text { Hairless } \\
\text { rat skin }{ }^{d)}\end{array} \\
\begin{array}{c}R_{\text {skin }} \\
(\mathrm{h} / \mathrm{cm})\end{array}\end{array}$} \\
\hline & $\begin{array}{c}L_{1} \\
(\mu \mathrm{m})\end{array}$ & $\begin{array}{c}C_{1}{ }^{\prime} \\
\left(\mathrm{mg} / \mathrm{cm}^{3}\right)\end{array}$ & $\begin{array}{c}C_{\mathrm{S}} \\
\left(\mathrm{mg} / \mathrm{cm}^{3}\right)\end{array}$ & $\begin{array}{c}D_{1} \\
\left(\mathrm{~cm}^{2} / \mathrm{h}\right)\end{array}$ & $\begin{array}{c}L_{2} \\
(\mu \mathrm{m})\end{array}$ & $K_{2 / 1}$ & $\begin{array}{c}D_{2} \\
\left(\mathrm{~cm}^{2} / \mathrm{h}\right)\end{array}$ & $\begin{array}{c}R_{2} \\
(\mathrm{~h} / \mathrm{cm})\end{array}$ & $\begin{array}{c}L_{3} \\
(\mu \mathrm{m})\end{array}$ & $K_{3 / 2}$ & $\begin{array}{c}D_{3} \\
\left(\mathrm{~cm}^{2} / \mathrm{h}\right)\end{array}$ & $\begin{array}{c}R_{3} \\
(\mathrm{~h} / \mathrm{cm})\end{array}$ & \\
\hline 0 & 65 & 4.86 & 0.722 & $3.91 \times 10^{-6}$ & 50 & 3.10 & $2.71 \times 10^{-6}$ & 595 & 65 & 0.323 & $3.91 \times 10^{-6}$ & 1662 & 336 \\
\hline 5 & 65 & 5.42 & 1.18 & $6.52 \times 10^{-6}$ & 50 & 1.39 & $4.56 \times 10^{-6}$ & 789 & 65 & 0.719 & $6.52 \times 10^{-6}$ & 997 & 590 \\
\hline 10 & 65 & 5.18 & 2.20 & $1.67 \times 10^{-5}$ & 50 & 0.838 & $1.07 \times 10^{-5}$ & 578 & 65 & 1.19 & $1.67 \times 10^{-5}$ & 398 & 769 \\
\hline 20 & 65 & 5.29 & 2.98 & $4.02 \times 10^{-5}$ & 50 & 0.329 & $3.69 \times 10^{-5}$ & 412 & 65 & 3.04 & $4.02 \times 10^{-5}$ & 162 & 829 \\
\hline
\end{tabular}

All parameters were calculated using the mathematical model describing the release of DHE from three types of the PSA tape illustrated in Fig. 2. $L_{1}, L_{2}, L_{3}$, thickness of the PSA layer, the EVA membrane and the adhesive layer, respectively, $C_{1}^{\prime}$, undissolved DHE concentration in the PSA layer, $C_{\mathrm{S}}$, dissolved DHE concentration in the PSA tape, $D_{1}, D_{2}$, $D_{3}$, diffusivity of DHE in the each layer; $K_{2 / 1}, K_{3 / 2}$, partition coefficient of DHE between layers, $R_{2}, R_{3}, R_{\text {skin }}$, permeation resistance of DHE in the each layer and the excised skin of hairless rat. a) The cumulative amount of DHE released from the PSA tape, Type I, was fitted to Eq. 7 using fixed $L_{1}$ and $C_{\mathrm{S}}$, and thereby initial $C_{1}^{\prime}$ and $D_{1}$ were calculated. $b$ ) The cumulative amount of DHE released from the PSA tape with EVA membrane, Type II, was fitted to Eq. 7 using fixed $C_{\mathrm{S}}$, initial $C_{1}^{\prime}, L_{1}, L_{2}$ and $D_{1}$, and thereby $K_{2 / 1}$ and $D_{2}$ were estimated. $R_{2}$ was calculated using Eq. 8. c) Supposing that the adhesive layer was composed of the same element as the PSA layer, $D_{3}$ and $K_{3 / 2}$ were substituted by $D_{1}$ and the inverse of $K_{2 / 1} . R_{3}$ was calculated using Eq. 9. d) $R_{\text {skin }}$ was calculated by Eq. 10 using $C_{\mathrm{S}}$ in the PSA layer and the steady-state flux of DHE in the in-vitro permeation study which is shown in Fig. 4.

A) Type 1

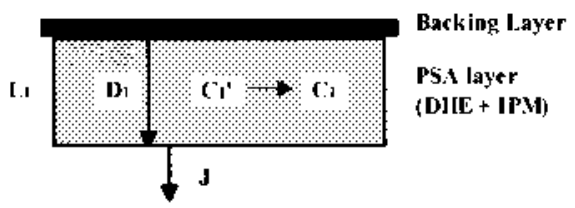

Bi) lype II

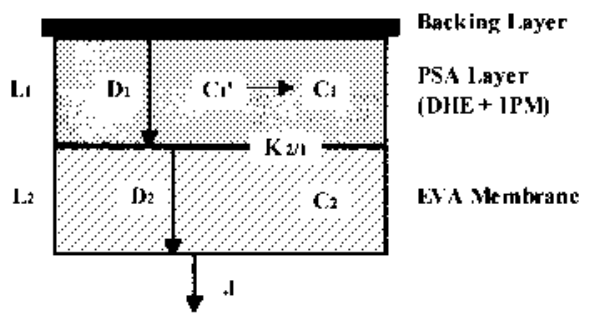

C) Type ill

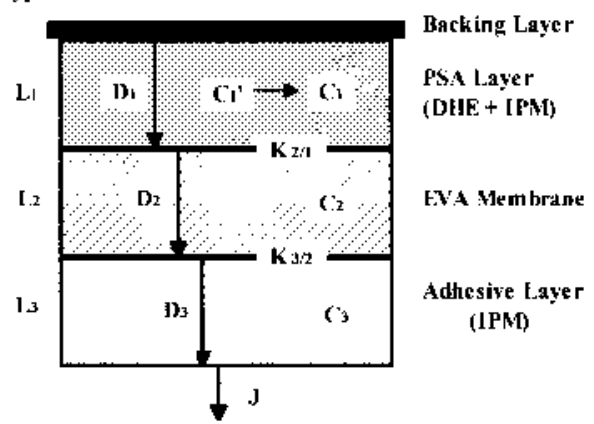

Fig. 2. Schemes of the Three Types of the PSA Tape, Which Was Investigated in This Study, and of the Mathematical Model for the Release of DHE from the PSA Tape

A) Type I is a mono-layer tape as the PSA layer contained DHE. B) Type II is a bilayer tape as the PSA layer attached with the EVA membrane. C) Type III is a tri-layer tape as the PSA tape attached with the EVA membrane and the adhesive layer. $C_{1}, C_{2}$, $C_{3}$, dissolved DHE concentration in the PSA layer, the EVA membrane and the adhesive layer, respectively, $C_{1}{ }^{\prime}$, undissolved DHE concentration in the PSA layer, $L_{1}, L_{2}, L_{3}$, thickness of the each layer, $D_{1}, D_{2}, D_{3}$, diffusivity of DHE in the each layer; $K_{2 / 1}, K_{3 / 2}$, partition coefficient of DHE between layers, $J$, release rate of DHE from each type of tape.

the Skin The steady-state flux of DHE through hairless rat skin during the application of the PSA tape is shown in Fig. 4. The flux of DHE was enhanced with the content of IPM in the PSA tape, and it was at a maximum in the range from 20 to $40 \%$ of IPM. When the tape containing in excess of $20 \%$

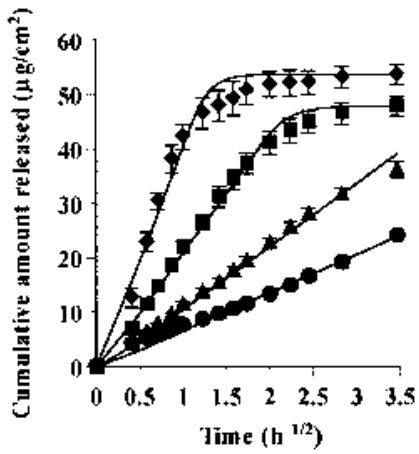

Fig. 3. The Cumulative Amount of DHE Released from the PSA Tape, Type I, Containing $0(\bullet), 5(\boldsymbol{\Delta}), 10(\mathbf{\square})$ and $20 \%(\bullet)$ of IPM

Each point represents the mean and standard deviation $(n=4)$. Solid lines indicate the calculated value from the mathematical model (Fig. 2A).

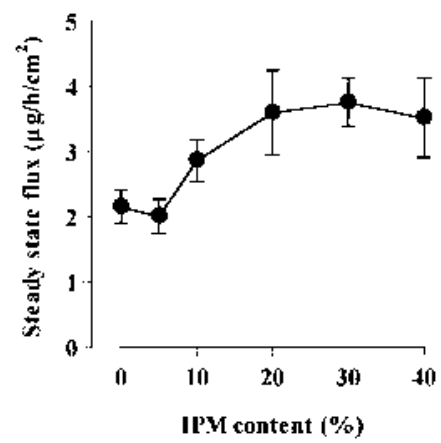

Fig. 4. The Steady-State Flux of DHE through Hairless Rat Skin during Application of the PSA Tape, Type I, Containing Various Concentrations of IPM

Each point represents the mean and standard deviation $(n=4)$.

of IPM was removed after the application onto the skin for $24 \mathrm{~h}$, however, it was found that a part of PSA layer remained on the surface of the skin. Therefore, an IPM content of $10 \%$ in the PSA tape was preferred, which enhanced 2-fold the DHE permeation level observed without the IPM.

Permeation of DHE through the EVA Membrane DHE was permeable to the EVA membrane (Fig. 5). The flux of DHE was enhanced with the content of vinyl acetate in the EVA membrane, and a semilogarithmical linearity was found as reported for several drugs. ${ }^{13,21)}$ DHE had a mild lipophilic- 


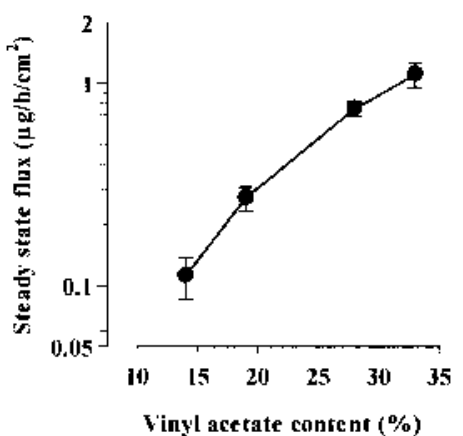

Fig. 5. The Steady-State Flux of DHE through the EVA Membrane with Various Contents of Vinyl Acetate

Each point represents the mean and standard deviation $(n=4)$.

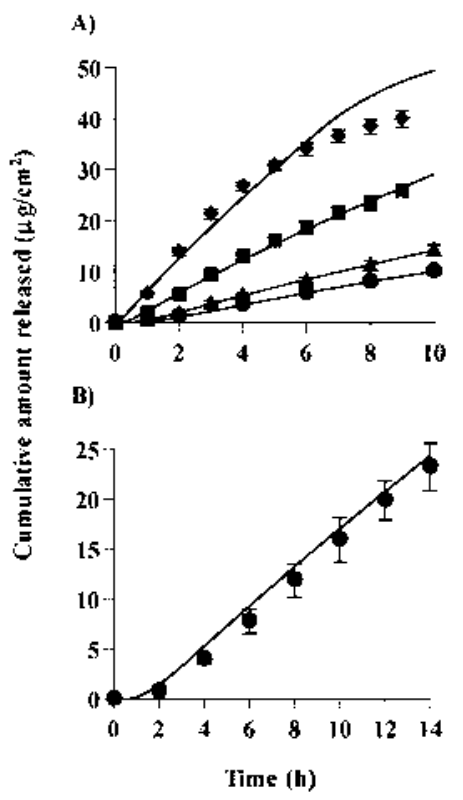

Fig. 6. The Cumulative Amount of DHE Released from the PSA Tape with the EVA Membrane and the Adhesive Layer, Type II and III

A) Type II, contained $0(\bullet), 5(\mathbf{\Lambda}), 10(\mathbf{\square})$ and $20 \%(\bullet)$ of IPM in the PSA layer. Solid lines indicate the calculated values from the mathematical model (Fig. 2B). B) Type III, contained $10 \%$ of IPM in the PSA layer. Solid lines indicate the calculated values from the mathematical model (Fig. 2C). Each point represents the mean and standard deviation $(n=4)$

ity $\left.(\log P, 2.13)^{5}\right)$ and the solubility parameter of DHE calculated by Fedors's method ${ }^{22)}$ was $12.09\left(\mathrm{cal} / \mathrm{cm}^{3}\right)^{1 / 2}$, which was suitable to control the permeability by vinyl acetate content in the membrane. ${ }^{21)}$ However, in the 28 and $33 \%$ of vinyl acetate containing EVA membranes it was hard to maintain the identical thickness of membrane during the attachment to the PSA tape, because these membrane were too soft. Therefore, $19 \%$ of vinyl acetate containing EVA membrane was chosen as the release controlled membrane for this formulation.

Release of DHE from the PSA Tape with the EVA Membrane, Type II and III The DHE was released from the PSA with the EVA membrane, Type II, in accordance with zero-order rate, and the release rate increased with the IPM content in the PSA layer (Fig. 6A). Calculated parameters, $K_{2 / 1}$ and $D_{2}$, by fitting-calculation did not depend on their initial values. As the result of the calculation, the increase of IPM content in the PSA layer increased the diffusivity of DHE in the EVA membrane, $D_{2}$, and decreased the partition ratio of DHE in the EVA membrane to the PSA

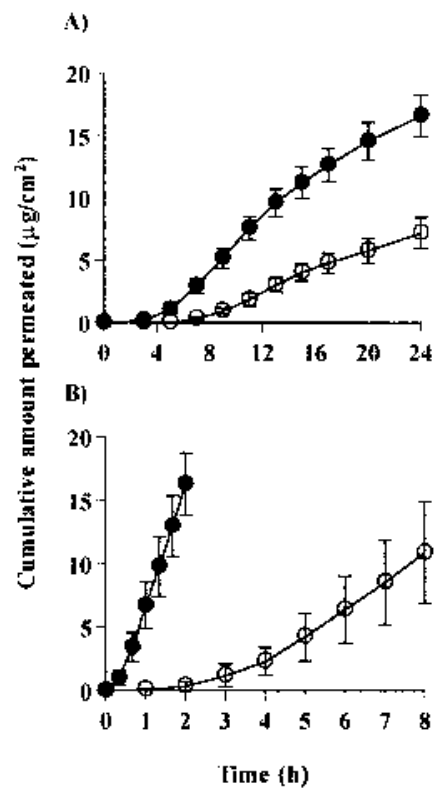

Fig. 7. The Cumulative Amount of DHE Permeated through Hairless Rat Skin with $(\bullet)$ and without $(O)$ SC Damage after the Application of the PSA Tape with (A, Type III) and without (B, Type I) the EVA Membrane Contained $10 \%$ IPM

The data presented in Fig. 7B are referenced from our previous report (ref. 6). Each point represents the mean and standard deviation $(n=4)$.

layer, $K_{2 / 1}$ (Table 1). Therefore, the permeation resistance of DHE in the EVA membrane, $R_{2}$, was not affected by the IPM content. The release rate from the Type II tape with $20 \%$ of IPM gradually decreased (Fig. 6A). This might be caused by the gradual increase in the resistance of DHE due to the IPM in the PSA layer transferred to the EVA membrane with time.

When using the PSA tape with the EVA membrane and adhesive layer, Type III, of which the adhesive layer was composed of the same element as the PSA layer, the permeation resistance of DHE in the adhesive layer, $R_{3}$, appeared to be markedly influenced by the IPM content in the adhesive layer (Table 1). In the case of 10\% IPM contained adhesive layer, the total permeation resistance of DHE, the sum of $R_{2}$ and $R_{3}$, was $976 \mathrm{~h} / \mathrm{cm}$, which was somewhat larger than the permeation resistance in hairless rat skin when using the Type I tape with $10 \%$ IPM, $769 \mathrm{~h} / \mathrm{cm}$. In this case, the cumulative amount profile of released DHE could be estimated satisfactorily and indicated about a 2-h lag-time and $1.85 \mu \mathrm{g} / \mathrm{h} / \mathrm{cm}^{2}$ of steady-state release rate (Fig. 6B). It was demonstrated that the Type III tape including an adequate content of IPM, which was estimated from kinetic analysis, could control the release of DHE similarly to the permeation through the skin.

Permeation through the Skin Applied by the PSA Tape The cumulative amount of permeated DHE through the skin after application of the PSA tape with and without the EVA membrane, Type III and I, are shown in Fig. 7. When the PSA tape with the EVA membrane, Type III, was applied onto the SC damaged skin, the steady-state flux of DHE $0.93 \mu \mathrm{g} / \mathrm{h} / \mathrm{cm}^{2}$, was not 2-fold higher than that of non-damaged skin, $0.47 \mu \mathrm{g} / \mathrm{h} / \mathrm{cm}^{2}$ (Fig. 7A). On the contrary, when using the PSA tape without the EVA membrane, Type I, the steady-state flux of DHE through the damaged skin, 9.66 $\mu \mathrm{g} / \mathrm{h} / \mathrm{cm}^{2}$, was 4.5 -fold higher than that of non-damaged skin, $2.15 \mu \mathrm{g} / \mathrm{h} / \mathrm{cm}^{2}$ (Fig. 7B). $\left.{ }^{6}\right)$ These results indicate that 


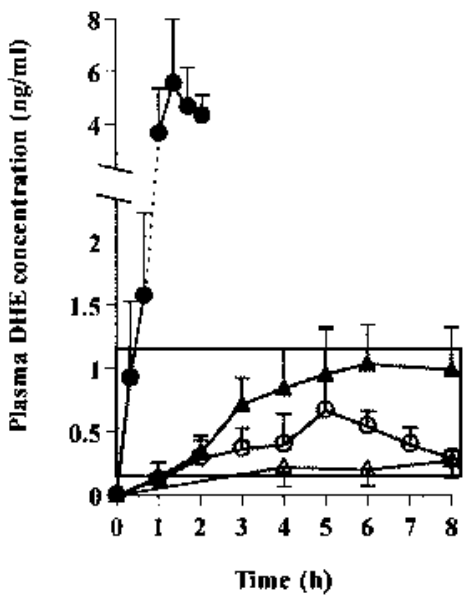

Fig. 8. The Plasma DHE Concentration during the Application of the PSA Tape with $\left(0.95 \mathrm{~cm}^{2}, \boldsymbol{\Delta} \triangle\right)$ and without $\left(0.28 \mathrm{~cm}^{2}, \boldsymbol{\bigcirc}\right)$ the EVA Membrane, Type III and I, onto the SC Damaged Skin (Closed Symbols) or Non-damaged Skin (Open Symbols) in Hairless Rats

The shaded area indicates the pharmacologically effective concentration in plasma as previously reported (ref. 6). Each point represents the mean and standard deviation $(n=4)$.

Type III is possible to prevent an excessive increase of DHE permeation due to SC damage. However, there were somewhat large lag-times, 4 and $6 \mathrm{~h}$, these are undesirable during an initial dose. In the case of analgesic therapy, it has been reported that a withdrawal time is necessary to reduce the tolerance to analgesic tolerance. ${ }^{23)}$

Plasma Concentration Following the Application of the PSA Tape The plasma DHE concentrations following application of the PSA tape with and without the EVA membrane, Type III and I, onto the abdominal surface of hairless rats are shown in Fig. 8. When the PSA tape without the EVA membrane, Type I $\left(0.28 \mathrm{~cm}^{2}\right)$, was applied onto the skin of rats without the SC damage, the plasma DHE concentration was maintained between 0.2 and $0.7 \mathrm{ng} / \mathrm{ml}$ for $8 \mathrm{~h}$, which is in the range of the pharmacologically effective concentration, $0.2-1.2 \mathrm{ng} / \mathrm{ml}$ (shaded area in Fig. 8) reported previously. ${ }^{6}$ In the case of application onto the SC damaged skin, however, the concentration rose promptly and reached $5.50 \mathrm{ng} / \mathrm{ml}$, which is apparently in the toxic concentration range. Therefore, the rats had been severely anesthetized and depressed respiration, and some of them died after 3-h application.

On the other hand, when the PSA tape with the EVA membrane, Type III $\left(0.95 \mathrm{~cm}^{2}\right)$, was applied onto the skin of rats with the SC damage, the DHE concentration was maintained at $0.2-1.1 \mathrm{ng} / \mathrm{ml}$ for $8 \mathrm{~h}$. When it was applied onto the non-damaged skin, the DHE concentration was maintained at $0.2-0.3 \mathrm{ng} / \mathrm{ml}$ from 4 to $8 \mathrm{~h}$. This result indicates that Type III is able to prevent the unexpected increase of DHE concentration and the expression of toxic effect by SC damage.

In conclusion, we prepared PSA tape containing the novel opioid analgesic DHE with a diffusion-controlling EVA membrane, which was able to prevent from the unpredictable increase in skin permeation of DHE caused by SC damage. However, the controlled release profiles demonstrated in this study were only found in the case that the PSA tape was attached with the EVA membrane immediately before its topical application. For instance, it is anticipated that an easy-re- movable liner between the PSA tape and the EVA membrane permits the preparation of this formulation. If the PSA tape and the EVA membrane had been attached together previously, a burst of DHE release would occur after the application onto SC damaged skin. ${ }^{12)}$ Therefore, it is suitable that the PSA tape of DHE is attached to the EVA membrane immediately before application.

It is reported that the sublingual dose of DHE relieved $90 \%$ of cancer patient from the moderate and severe pain, but the clinical side effects were found, which were mainly dizziness $(72 \%)$, somnolence $(60 \%)$, nausea $(30 \%)$, vomiting $(16.5 \%)$, constipation $(5 \%)$ and shortness of breath $(8 \%){ }^{24)}$ Although there is no report regarding the DHE concentration in human plasma, the therapeutic concentration range of DHE is probably narrow. If the therapeutic concentration range, the body-clearance of DHE and the permeation resistance of DHE through the human skin are informed, the PSA tape with the EVA membrane is most suitable for clinical therapy will be formulated. As the results in this study, it is possible that the PSA tape with EVA membrane can prevent the clinical side effect of DHE by the undesirable increase of skin permeation.

\section{REFERENCES}

1) Wang D. X., Lu X. Q., Qin B. Y., J. Pharm. Pharmacol., 47, 669-673 (1995).

2) Bentley K. W., Hardy D. G., J. Am. Chem. Soc., 89, 3281-3292 (1967).

3) Tokuyama S., Nakamura F., Takahashi M., Kaneto H., Biol. Pharm. Bull., 19, 477-479 (1996).

4) Aceto M. D., Harris L. S., Bowman E. R., Eur. J. Pharmacol., 338, $215-223$ (1997)

5) Ohmori S., Hayashi T., Kawase M., Saito S., Morimoto Y., J. Pharmacol. Exp. Ther., in press, (2001).

6) Ohmori S., Hayashi T., Kawase M., Saito S., Sugibayashi K., Morimoto Y., J. Pharm. Pharmacol., in press, (2001).

7) Franz T. J., Tojo K., Shah K. R., Kydonieus A., "Treatise on Controlled Drug Delivery: Fundamental, Optimization, Applications," ed. by Kydonieus A., Marcel Dekker, New York, 1992, pp. 341- 421.

8) Bronaugh R. L., Stewart R. F., J. Pharm. Sci., 74, 1062-1066 (1985).

9) Yoshiike T., Aikawa Y., Sindhvananda J., Suto H., Nishimura K., Kawamoto T., Ogawa H., J. Dermatol. Sci., 5, 92-96 (1993).

10) Chiaretti A., Schembri W. D., Tortorolo L., Piastra M., Polidori G., Acta Paediatr., 86, 330-331 (1997).

11) Benfeldt E., Seruo J., Arch. Dermatol. Res., 291, 517-526 (1999).

12) Seki T., Kawaguchi T., Sugibayashi K., Juni K., Morimoto Y., Chem. Pharm. Bull., 38, 740-743 (1990).

13) Chen G. S., Kim D. D., Chien Y. W., J. Control. Release, 34, 129-143 (1995).

14) Altenburger R., Rohr U. D., Kissel T., Pharm. Res., 15, 1238-1243 (1998).

15) Baker R. W., Heller J., "Transdermal drug delivery," ed. by Hardgraft J., Guy R. H., Marcel Dekker, New York, 1989, pp. 293-311.

16) Barber R. B., Rapoport H., J. Med. Chem., 18, 1074-1077 (1975).

17) Aitken-Nichol C., Zhang F., McGinity J. W., Pharm. Res., 13, 804 808 (1996).

18) Ohmori S., Hayashi T., Kawase M., Saito S., Morimoto Y., J. Chromatogr. B, 740, 253-263 (2000).

19) Higuchi T., J. Pharm. Sci., 50, 874-875 (1961).

20) Crank J. (ed.), "The Mathematics of Diffusion, Second Edition," Oxford University Press, London, 1975.

21) Kajimoto H., Sugibayashi K., Morimoto Y., J. Pharm. Sci. Technol. Jpn., 55, 127-133 (1995).

22) Fedors R. F., Polym. Eng. Sci., 14, 147-154 (1974).

23) Ouellet D. M.-C., Pollack G. M., J. Pharmacol. Exp. Ther., 281, 713720 (1997).

24) Wu G. Q., Sun Y., Chin. J. Oncol., 13, 64-67 (1991). 